\title{
A Review of Bioinspired Vision Sensors and Their Applications
}

\author{
Dong-il "Dan" Cho 1,2,3,* and Tae-jae Lee ${ }^{1,3}$ \\ ${ }^{1}$ Automation and Systems Research Institute (ASRI), Seoul National University, \\ Gwanakro 1, Gwanak-Gu, Seoul 151-742, Korea \\ ${ }^{2}$ Inter-university Semiconductor Research Center (ISRC), Seoul National University, \\ Gwanakro 1, Gwanak-Gu, Seoul 151-742, Korea \\ ${ }^{3}$ Department of Electrical and Computer Engineering, Seoul National University, \\ Gwanakro 1, Gwanak-Gu, Seoul 151-742, Korea
}

(Received December 18, 2014; accepted April 23, 2015)

Key words: $\quad$ bioinspired, bionic, vision sensor, computer vision, robotics

Bioinspired vision sensors have become very attractive in recent years because of their inherent redundancy suppression, integrated processing, fast sensing capability, wide dynamic range, and low power consumption. These sensors combine functionalities of the biological "where" and "what" systems of the human visual system and process the visual information using an asynchronous event-driven method. Since the emergence of bioinspired vision sensors, various applications based on them have been proposed in the computer vision and robotics fields. In this paper, we review bioinspired vision sensors and their applications. The reviewed sensors include dynamic vision sensors (DVSs), asynchronous time-based image sensors (ATISs), and dynamic and active pixel vision sensors (DAVISs). The reviewed applications based on the bioinspired vision sensors include visual tracking, detection, recognition, simultaneous localization and mapping (SLAM), reconstruction, stereo matching, and control.

\section{Introduction}

Until recently, charge-coupled devices $(\mathrm{CCDs})^{(1,2)}$ or complementary metal-oxide silicon $(\mathrm{CMOS})^{(3,4)}$ image sensors along with digital signal processing units that execute computer vision algorithms have been used for realizing conventional vision systems such as those for object tracking, object recognition, 3D reconstruction, simultaneous localization and mapping (SLAM), and navigation. These devices obtain visual information in the form of time-quantized "snapshots" recorded at a predetermined frame rate. During the period of transition from frame to frame, a problem similar to the undersampling phenomenon may arise. ${ }^{(5)}$ This shortcoming may be tolerable for

"Corresponding author: e-mail: dicho@snu.ac.kr 
a human observer, but artificial vision systems that require real-time processing, such as autonomous robot navigation or high-speed control, may fail as a consequence of this shortcoming. Another problem of frame-based visual information acquisition is redundancy. Each recorded frame displays the information from all pixels, regardless of whether this information has changed since the last frame. This method obviously leads to a high degree of redundancy in the acquired image data. Obtaining and processing these unnecessary data waste resources and lead to increased channel bandwidth requirements, high transmission power dissipation, and increased memory size.

In contrast, biological sensing systems operate in a different way. In nature, including in human beings, the sensing systems of organisms are acted upon by asynchronous events, and the information is processed hierarchically and in parallel in a massive neuron network. ${ }^{(6)}$ This idea has stimulated numerous studies to understand the relationship between the human sensory and central nervous systems, and to apply the knowledge of computational neuroscience to construct intelligent machines. It also gave rise to a new field called neuromorphic engineering in the late $1980 \mathrm{~s} .{ }^{(7,8)}$

In neuromorphic vision devices, various sensors have been developed over the past two decades, including temporal contrast vision sensors, gradient-based sensors, edge-orientation sensitive sensors, and optical flow sensors. ${ }^{(9-11)}$ However, very few have thus far been used in practical applications. Many lack technical completeness because of circuit complexity, large pixel area or high noise level, preventing realistic application. ${ }^{(5)}$ More recently, however, there have been many improvements in vision sensors based on biological principles in terms of performance and practicality. These sensors include dynamic vision sensors (DVSs), ${ }^{(12,13)}$ asynchronous time-based image sensors (ATISs), ${ }^{(14-16)}$ and very recently developed dynamic and active pixel vision sensors (DAVISs). ${ }^{(17,18)}$

In this paper, we review bioinspired vision sensors and their applications in the computer vision and robotics fields. The paper is organized as follows. In $\S 2$, reviews of the biological vision system and bioinspired vision sensors are presented. In $\S 3$, reviews of various applications and algorithms based on the bioinspired vision sensors are given. In $\S 4$, conclusions are shown.

\section{Bioinspired Vision Sensors}

\subsection{Biological vision system}

Prior to reviewing bioinspired vision sensors, it is necessary to look at biological vision systems, especially the human retinal system. The human retinal system is composed of photoreceptors, bipolar cells, and ganglion cells. ${ }^{(19,20)}$ Within the retina, photoreceptors are connected to horizontal cells, which are then connected to bipolar cells. Once the light enters the eye and passes through the eye lens, it is detected by photoreceptors that convert light into electrical pulses. These pulses are reflected and pass through bipolar and ganglion cells to enter optical fibers. Vision is realized after the visual cortex recognizes the electrical pulses. A schematic drawing of the retina network is illustrated in Fig. 1.

The translation of light into visual information initiates from two types of ganglion cells, X- and Y-cells. X-cells are distributed along the Parvo-cellular pathway that 


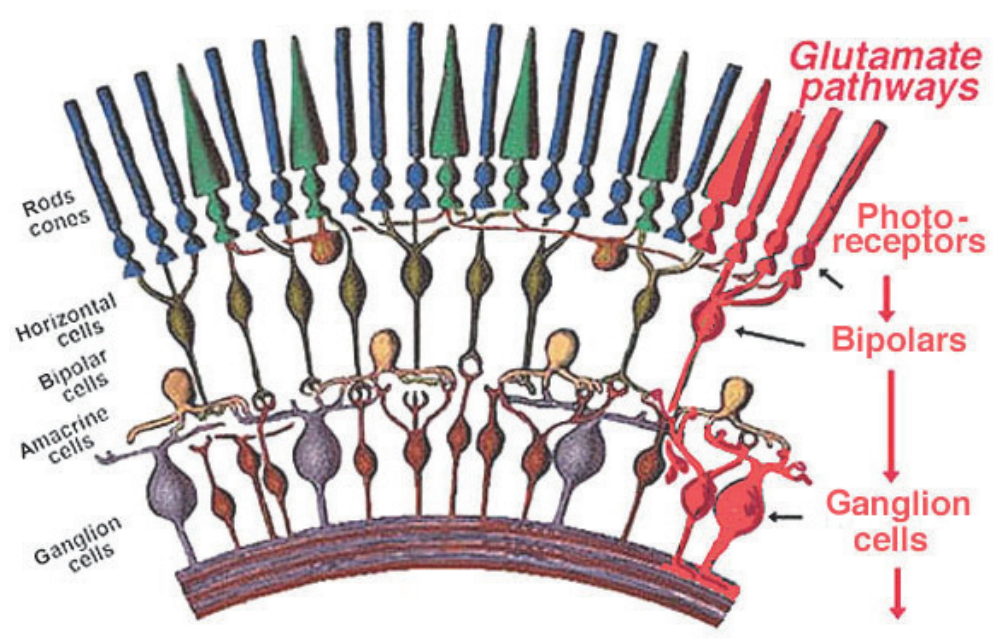

Fig. 1. (Color online) Schematic drawing of the retina network. ${ }^{(19)}$

occupies $80 \%$ of the nerve fibers and are focused on the fovea. The Parvo-cellular pathway usually carries color information, spatial details, and patterns. For this reason, the Parvo-cellular system is often referred to as the biological "what" system. On the other hand, Y-cells are distributed along the Magno-cellular pathway that occupies only $10 \%$ of the nerve fibers. The Magno-cellular pathway usually carries visual information related to changes, including the detection of the movement, distance, and speed of an object. Owing to this, the Magno-cellular system is often referred to as the biological "where" system.

\subsection{DVS}

Conventional frame-based image sensors suffer from data redundancy and dataprocessing delay problems. There have been many attempts to resolve these problems. Several event-based (frame-free) temporal contrast vision sensors have been reported in recent years. ${ }^{(21,22)}$ However, the sensor proposed by Kramer ${ }^{(21)}$ had low contrast sensitivity, whereas that proposed by Zaghloul and Boahen ${ }^{(22)}$ suffered from poor fixed pattern noise (FPN). Lichtsteiner et al. presented the first practical so-called DVS that mimics the function of Y-cells in an attempt to sense the dynamic information of the scene. ${ }^{(12,13)}$ The sensor is "event-driven" instead of clock-driven, and similarly to its biological model, it responds to "natural" events occurring in the scene it observes. The pixel autonomously responds to relative changes in intensity at a microsecond temporal resolution. Each pixel asynchronously sends out an ON event if the log-compressed light intensity of the pixel increases by a fixed amount and an OFF event when it decreases. This way, information is continuously transmitted and processed, and communication bandwidth is only used by active pixels. This type of asynchronous event-based data format is called address event representation (AER) protocol and was introduced by 
Sivilotti and Mahowald to model the transmission of neural information within biological systems. ${ }^{(23,24)}$ The sensor has a low FPN (2.1\%), a low power consumption $(24 \mathrm{~mW})$, a small pixel array size $(128 \times 128)$, a low latency $(15 \mu \mathrm{s})$, and a high dynamic range $(120$ $\mathrm{dB})$. The DVS is the first commercially available product belonging to a neuromorphic sensor class.

The picture and design of the DVS are shown in Fig. 2. ${ }^{(12)}$ The DVS pixel is composed of a fast logarithmic photoreceptor, a differencing circuit, and two comparators. In the logarithmic photoreceptor circuit, the photocurrent of a photodiode is sourced by a saturated N-channel metal-oxide-semiconductor field-effect transistor (MOSFET). The gate of the N-channel MOSFET is connected to the output of an inverting amplifier, which has the structure of a cascaded common source amplifier. Attributed to this transimpedance configuration, the photocurrent is logarithmically converted to the voltage $V_{\mathrm{p}}$. In the differencing circuit, two capacitors are integrated to amplify the signal. In addition, a reset switch is connected between the input and output ( $\left.V_{\text {diff }}\right)$ of the inverting amplifier to remove the DC mismatch. The comparators determine whether the status of events is ON or OFF using the level of $V_{\text {diff. }}$.

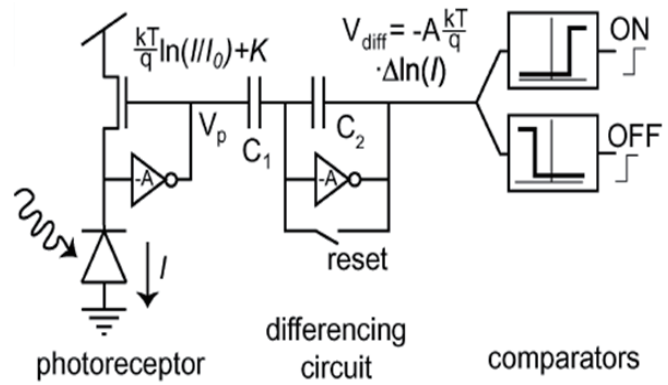

(a)

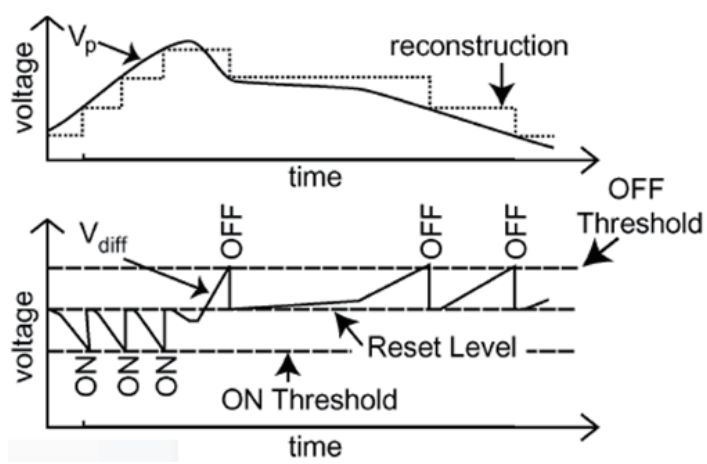

(b)

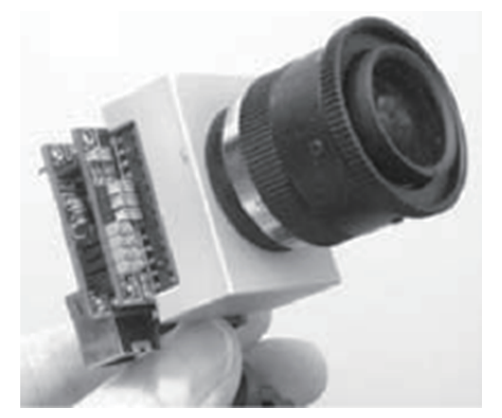

(c)

Fig. 2. (a) Abstracted pixel schematic. (b) Principle of operation. (c) DVS sensor. ${ }^{(12)}$ 


\subsection{ATIS}

In addition to the invention of the DVS, consideration has been given to creating a new type of sensor that combines the "where" and "what" systems. The so-called ATIS brings these systems together using numerous bioinspired approaches such as event-based imaging. ${ }^{(14-16)}$ This sensor is based on an array of fully autonomous pixels containing an event-based change detector (CD) and a pulse width modulation (PWM)-based exposure measurement (EM) unit. The EM unit is locally initiated by an individual pixel that detects a change in brightness in its field-of-view from the CD. The sensor outputs the temporal contrast event data and the absolute intensity of each event. The sensor has a very low FPN $(0.25 \%)$, a low power consumption $(<175 \mathrm{~mW})$, a reasonable pixel array size $(304 \times 240)$, a low latency $(4 \mu$ s $)$, and a high dynamic range (125 dB). The ATIS has not yet been commercialized.

The composition and operation of an ATIS pixel are illustrated in Fig. 3. The structure of the CD is similar to that of the DVS pixel. The EM circuit is composed of a photodiode, a capacitor, a P-channel MOSFET switch, and a comparator. The reset

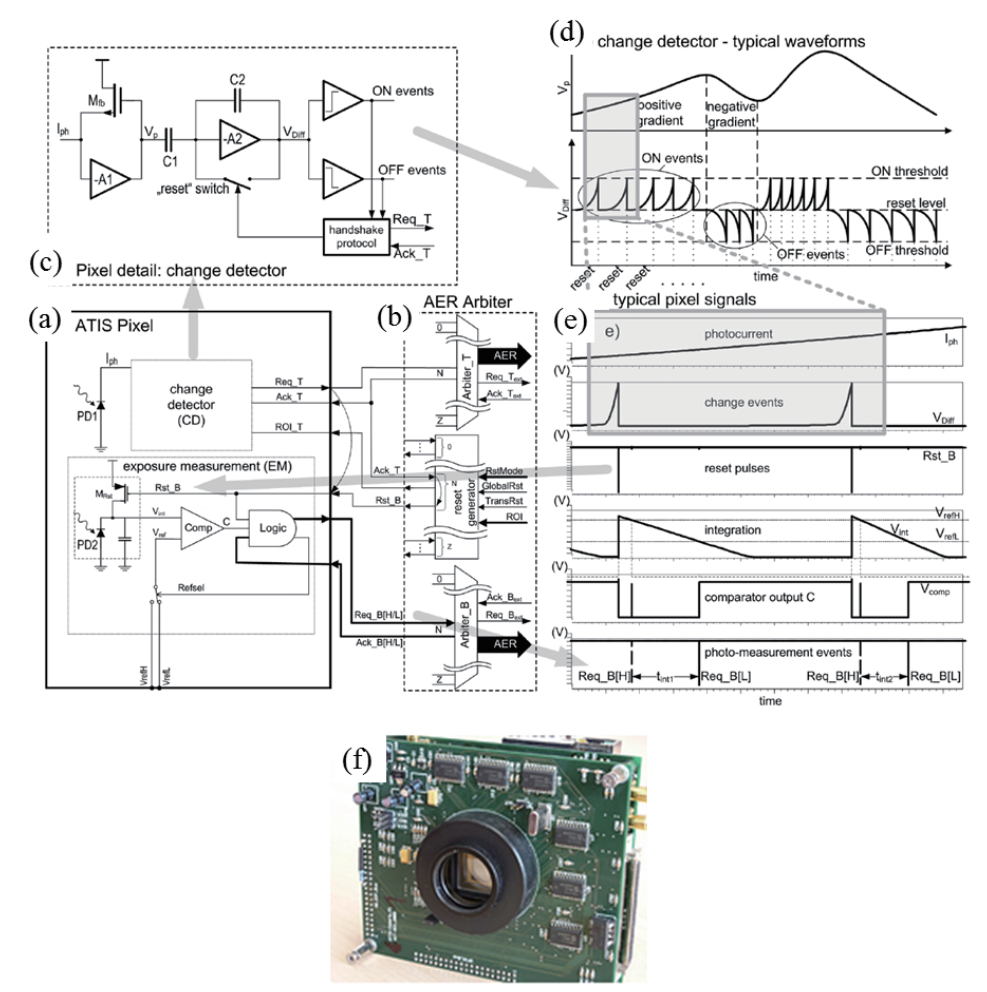

Fig. 3. (Color online) (a) ATIS pixel connected to (b) arbiters and reset generator/mode-ofoperation/ROI controller, (c) change detector schematic, (d) typical change detector signals, and (e) exemplary pixel waveforms illustrating two change event-triggered exposure measurement cycles. ${ }^{(15)}$ (f) Photograph of the ATIS. ${ }^{(25)}$ 
signal, generated from the change detector during the $\mathrm{ON}$ events, is applied to the gate of the P-channel MOSFET switch. Therefore, the capacitor is charged when the ON event occurs. After the reset, the capacitor voltage $\left(V_{i n t}\right)$ gradually decreases because the capacitor is discharged by the photocurrent generated in the photodiode. When the level of $V_{\text {int }}$ is lower than the slice level of the comparator $\left(V_{\text {ref }}\right)$, the comparator output $C$ is converted from a low signal to a high signal. Before the next $\mathrm{ON}$ event occurs, the high level of the comparator output $C$ is maintained. Because the decreasing rate of the level of $V_{\text {int }}$ is proportional to the intensity of light that illuminates the photodiode, the light intensity determines the duration of the comparator output $C$.

\subsection{DAVIS}

Very recently, a DAVIS has been proposed as another version of a combined biological "where" and "what" system.(17,18) The DAVIS combines the advantages of DVSs and active pixel sensors (APSs) at the pixel level. It outputs image frames through the synchronous APS pathway and simultaneously outputs events through the asynchronous DVS pathway. The shared photodiode (PD) and the small size of the APS circuit lead to a DAVIS pixel area that is $60 \%$ smaller than the ATIS pixel area. The sensor has a very low FPN $(0.5 \%)$, a very low power consumption $(<14 \mathrm{~mW})$, a reasonable pixel array size $(240 \times 180)$, a low latency $(3 \mu \mathrm{s})$, and a reasonable dynamic range (130 dB for DVS and $51 \mathrm{DB}$ for the frame image sensor). The DAVIS is also a commercially available product.

A schematic of the DAVIS pixel is shown in Fig. 4. A four-transistor CMOS APS, which is used to detect the light intensity, is integrated with the conventional DVS circuit. Using the photocurrent generated from the photodiode in the DVS circuit, the APS can obtain the readout light intensity. The detected information on the light intensity is stored in the parasitic gate capacitance of MN2. MN5, connected between the DVS and APS circuits, is used to reduce the reset transient of the source voltage of MN1.

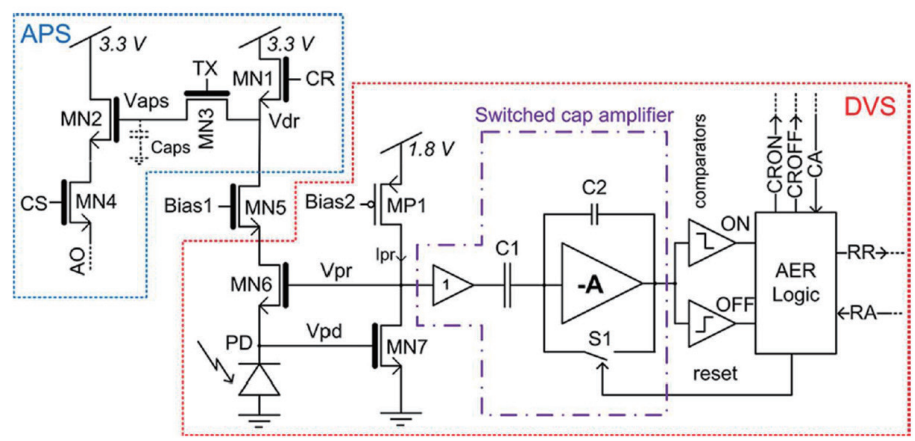

(a)

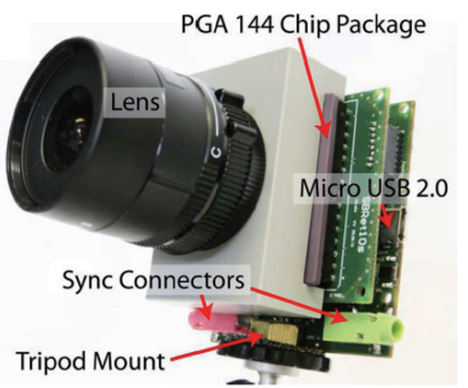

(b)

Fig. 4. (Color online) (a) DAVIS pixel schematic. (b) Photograph of the DAVIS.(17) 


\subsection{Summary of bioinspired vision sensors}

We reviewed the biological vision system and three bioinspired vision sensors. Table 1 shows the specifications of the previously reviewed bioinspired DVS, ATIS, and DAVIS. The DVS is a bioinspired "where" system that responds to relative changes. The ATIS is a combination of bioinspired "where" and "what" systems that contains event-based CD and PWM-based EM units. Both the DVS and the ATIS are based on an asynchronous event-driven method, and the single pixel handles its own visual information individually and autonomously. The DAVIS is a combination of an asynchronous "where" system and a synchronous "what" system. It outputs image frames through the synchronous APS pathway and simultaneously outputs events through the asynchronous DVS pathway.

With respect to the commercialization of bioinspired vision sensors, the DVS and DAVIS are already commercialized, while the ATIS has not yet been commercialized. While the ATIS and DAVIS are both biological "where" and "what' systems, the ATIS has several disadvantages over the DAVIS. The power consumption and pixel size of the ATIS are relatively high and large compared with those of the DAVIS. Furthermore, the ATIS only provides intensity measurements of event-detected pixels, while the DAVIS provides intensity measurements of all pixels. These disadvantages may make the ATIS difficult to be commercialized.

The reviewed bioinspired vision technologies have the potential to overcome the problems experienced in conventional vision-based systems, such as high power consumption and high computational load. Bioinspired vision sensors have already been applied to various computer vision and robotics applications. Detailed reviews will be presented in $\S 3$. In the future, much more progress in bioinspired vision sensors is expected.

Table 1

Specifications of bioinspired vision sensors.

\begin{tabular}{|c|c|c|c|}
\hline & $\operatorname{DVS}^{(12,13)}$ & ATIS(14-16) & DAVIS $^{(17,18)}$ \\
\hline Function & $\begin{array}{l}\text { Asynchronous temporal } \\
\text { contrast event detection }\end{array}$ & $\begin{array}{c}\text { Asynchronous temporal } \\
\text { contrast event detection } \\
+ \\
\text { Intensity measurement for } \\
\text { each event }\end{array}$ & $\begin{array}{c}\text { Asynchronous temporal } \\
\text { contrast event detection } \\
+ \\
\text { Synchronous image } \\
\text { capture }\end{array}$ \\
\hline First release year & 2006 & 2008 & 2013 \\
\hline Fixed pattern noise & $2.1 \%$ & $0.25 \%$ intensity & $0.5 \%$ APS, DVS 3.5\% \\
\hline Power consumption & $24 \mathrm{~mW}$ & $\begin{array}{c}175 \mathrm{~mW} \text { (high activity) } \\
50 \mathrm{~mW} \text { (low activity) }\end{array}$ & $\begin{array}{c}14 \mathrm{~mW} \text { (high activity) } \\
5 \mathrm{~mW} \text { (low activity) }\end{array}$ \\
\hline Array size & $128 \times 128$ & $304 \times 240$ & $240 \times 180$ \\
\hline Pixel size $\left(\mu \mathrm{m}^{2}\right)$ & $40 \times 40$ & $30 \times 30$ & $18.5 \times 18.5$ \\
\hline Latency & $15 \mu \mathrm{s} @ 1$ klux & $4 \mu \mathrm{s} @ 1$ klux & $3 \mu \mathrm{s} @ 1$ klux \\
\hline Dynamic range & $120 \mathrm{~dB}$ & $125 \mathrm{~dB}$ & $\begin{array}{c}130 \mathrm{~dB} \text { DVS } \\
51 \mathrm{~dB} \text { APS }\end{array}$ \\
\hline Commercialization & $\begin{array}{c}\text { Commercialized } \\
\text { (Product name: DVS128) }\end{array}$ & Not commercialized & $\begin{array}{c}\text { Commercialized } \\
\text { (Product name: DAVIS240) }\end{array}$ \\
\hline
\end{tabular}




\section{Applications of Bioinspired Vision Sensor}

In this section, various applications of bioinspired vision sensors are reviewed. Since the emergence of bioinspired vision sensors, various applications using these sensors have been proposed for computer vision and robotics. To the best of our knowledge, there have not yet been any applications proposed based on the DAVIS camera. There are a few applications based on the ATIS camera, but these applications do not utilize the absolute intensity information of each event. In the following, we will regard these cases as DVS camera-based applications. The reviewed applications can be classified into six categories: visual tracking, detection and recognition, SLAM, visual reconstruction, stereo matching, and control.

\subsection{Visual tracking}

Lizenberger et al. proposed an object tracking algorithm using a single DVS. ${ }^{(26)}$ The proposed algorithm was inspired by a conventional frame-based mean-shift approach and implements continuous clustering of address events and tracking of clusters. ${ }^{(27)}$ Figure 5 presents the people-tracking result obtained using the proposed method.

Lizenberger et al. presented a vehicle speed estimation algorithm for traffic monitoring based on a single DVS. ${ }^{(28)}$ The proposed method is able to measure the velocities of vehicles in the range of 20 to $300 \mathrm{~km} / \mathrm{h}$ on up to four lanes simultaneously.
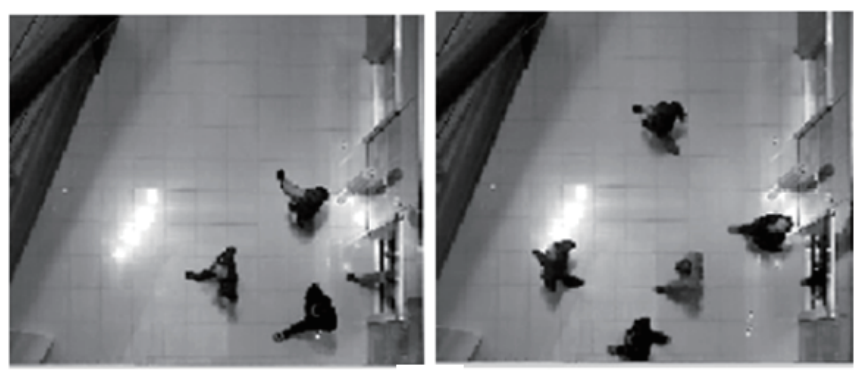

(a)
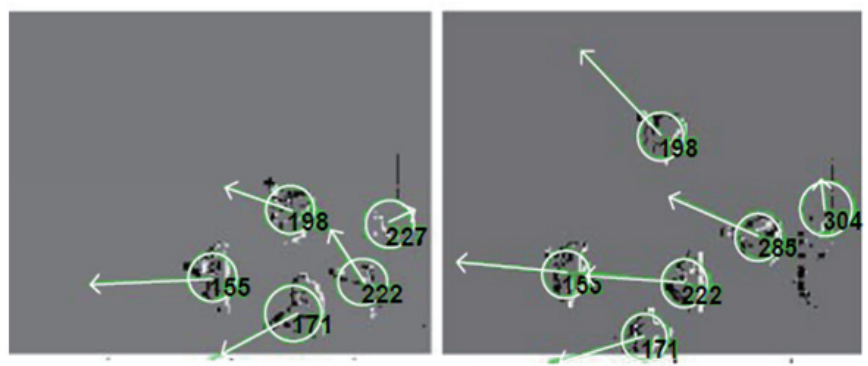

(b)

Fig. 5. (Color online) Examples of results for people tracking: (a) photograph of the experimental environment and (b) DVS-based tracking result. ${ }^{(26)}$ 
Bauer et al. further improved this system and proposed three different algorithms for vehicle speed estimation based on DVS output data stream processing. ${ }^{(29)}$

Benosman et al. proposed an optical flow algorithm for visual tracking using a single DVS. ${ }^{(30)}$ The optical flow estimate is obtained by adapting the differential flow brightness consistency constraint to an event-based domain. ${ }^{(31)}$ They further improved the optical flow estimation algorithm based on a local differential approach on the surface defined by events. ${ }^{(32)}$

\subsection{Detection and recognition}

Humenberger et al. proposed a stereo DVS-based fall detection application using a neural network for elderly people in a home environment. ${ }^{(33)}$ Using the stereo DVS camera system proposed in their previous work, ${ }^{(34)}$ a meaningful feature vector is calculated. The detailed explanation of feature extraction for fall detection can be found in another previous work of theirs. ${ }^{(35)}$ The neural network is used to classify the actual event as fall or non-fall. Figure 6 shows an example of a possible fall scenario.

Pérez-Carrasco et al. proposed a texture recognition hardware application based on convolutional neural networks. ${ }^{(36)}$ A monocular DVS camera and a 2D convolution chip combined with a host PC were used as the hardware setup. The proposed texture recognition method modified the conventional frame-based Manjunath's method to a frameless event-based sensing system. ${ }^{(37)}$ The experiments showed that the proposed recognition process can be achieved before the equivalent conventional frame-based system could capture and transmit the video while maintaining a similar recognition rate.
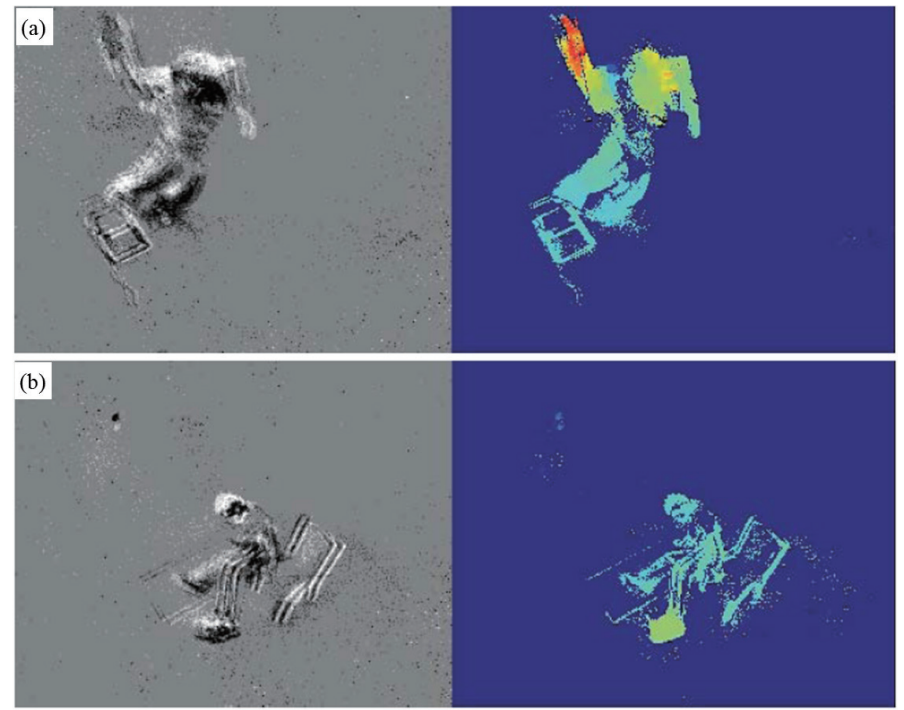

Fig. 6. (Color online) Examples of possible fall scenario. Left: address events from DVS and right: color-coded 3D representation after stereo matching. (a) Fall from ladder scenario. (b) Fall from chair scenario. ${ }^{(33)}$ 


\subsection{SLAM}

Weikersdorfer et al. proposed an upward-looking DVS-based 2D SLAM algorithm in an indoor environment. ${ }^{(38)}$ The previously proposed event-based feature tracking algorithm ${ }^{(39)}$ was used for landmark tracking. The experiments showed the feasibility of the proposed method in a small indoor environment. Figure 7 shows an example of a SLAM result obtained using the algorithm.

Mueggler et al. proposed an onboard quadrotor 6-degree-of-freedom (DOF) pose estimation system using a DVS camera that is able to track high-speed maneuvers such as flips. ${ }^{(40)}$ Their system starts by integrating events until a known artificial template is detected, and it then tracks the borders of the template by updating both line segments and the pose of the flying robot on an event-by-event basis. They demonstrated robust motion tracking during quadrotor flips with angular speeds up to $1200 \%$.

Because a DVS camera does not provide absolute brightness values, few attempts have been made to combine an event camera with an extra full frame camera. ${ }^{(41,42)}$ Weikersdorfer et al. developed an event-based 3D SLAM system combining a DVS with an RGB-D camera. ${ }^{(41)}$ The experiments showed that the proposed event-based 3D SLAM algorithm was twenty times faster than the conventional KinectFusion-based 3D SLAM. ${ }^{(43)}$

Similarly, Censi and Davide presented a low-latency event-based visual odometry system combining a DVS with a normal CMOS camera. ${ }^{(42)}$ The two sensors were automatically spatiotemporally calibrated on the basis of the computation of similarity statistics. Experiments showed that the rotation can be estimated with surprising accuracy, while the translation can be estimated only very noisily because it produces few events owing to a very small apparent motion.

\subsection{Visual reconstruction}

Kim et al. showed that an event stream with no additional sensing can be used to build a persistent and high-quality mosaic of a scene while a hand-held DVS camera is in rotational motion. ${ }^{(4)}$ Their method relies on two parallel probabilistic filters to
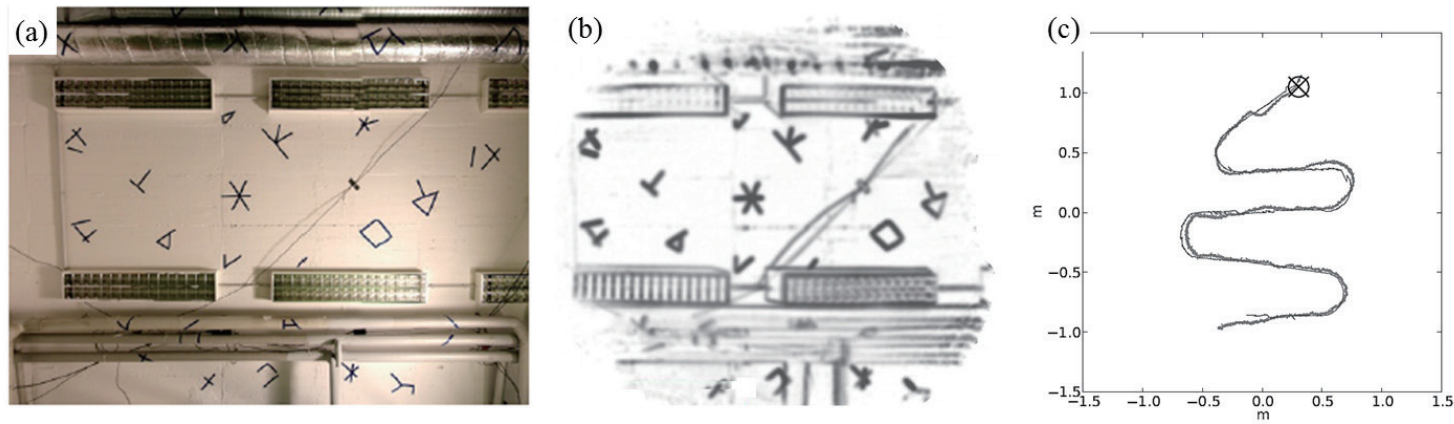

Fig. 7. (Color online) Experimental environment and result of SLAM. (a) Photograph of the ceiling. (b) Resulting map from SLAM algorithm. (c) Robot trajectory resulting from SLAM algorithm (gray) and external tracking system as a ground truth (black). ${ }^{(38)}$ 
jointly track the global rotational motion of a camera and estimate the gradients of the scene around it; the gradient map is then upgraded to a full image-like gray level mosaic with super-resolution and high dynamic range properties. Figure 8 shows a photograph of the experimental environment, DVS camera output, estimated gradient map, and reconstructed image-like mosaic of the scene.

Carneiro et al. proposed a 3D reconstruction algorithm of a moving object for more than two fixed DVS setups. ${ }^{(45)}$ A camera calibration method proposed by Benosman et $a l$. is used for multiple DVS calibration. ${ }^{(46)}$ After the calibration, geometrical and time constraints for matching events and Bayesian inference-based matching selection are used for the 3D reconstruction. Figure 9 shows examples of wireframe cube, human hand, and human face reconstruction results obtained using a 6 DVS camera setup.
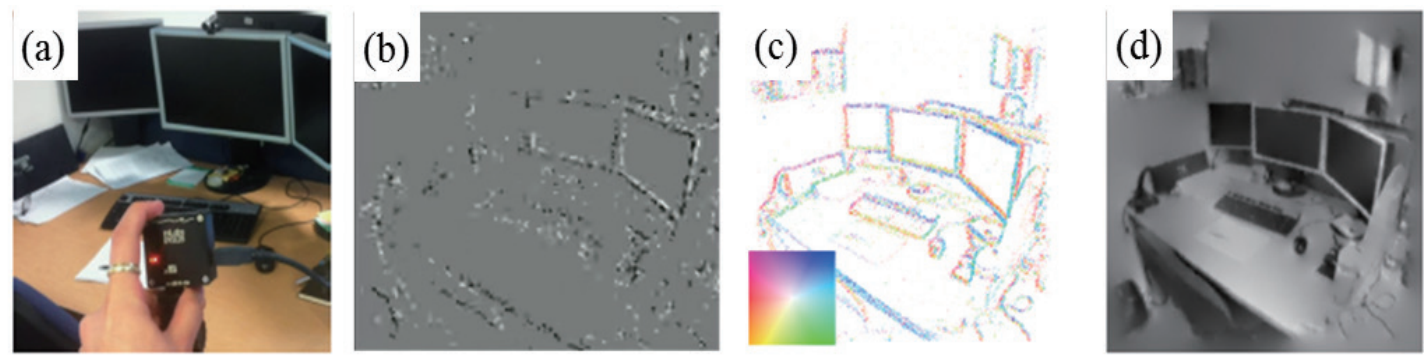

Fig. 8. (Color online) (a) Photograph of the experimental environment and a DVS camera. (b) DVS camera output. (c) Estimated gradient map. (d) Reconstructed image-like mosaic of the scene. ${ }^{(44)}$

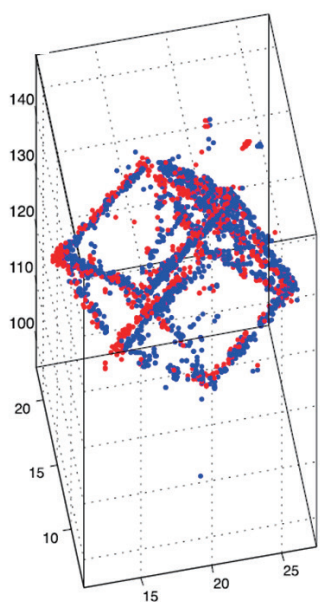

(a)

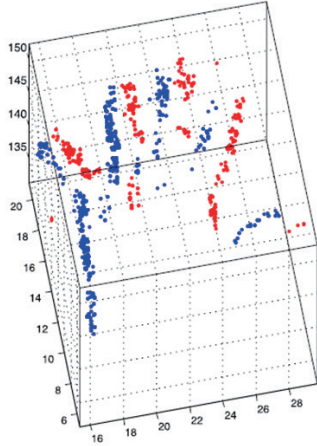

(b)

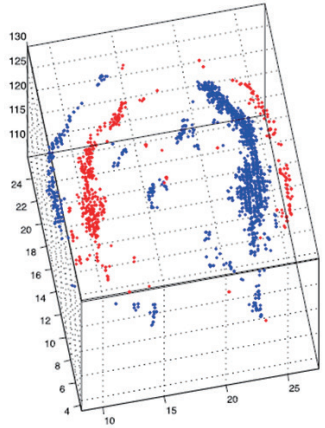

(c)

Fig. 9. (Color online) Example of reconstructions obtained using the 6 DVS camera system. (a) Wireframe cube, (b) hand, and (c) human face. The colors encode the polarities of the events producing the reconstructed 3D points. They give a hint to the motion's direction. ${ }^{(45)}$ 


\subsection{Stereo matching}

Various stereo matching algorithms for stereo DVS camera setups have been proposed.(47-50) Sulzbachner et al. proposed an address event frame, a collection of events over a defined time period, based on the correlation method for stereo matching algorithms. ${ }^{(47)}$ Rogister et al. proposed an asynchronous event-based binocular stereo matching algorithm combining epipolar geometry and timing information. ${ }^{(48)}$ Taking advantage of the high temporal resolution and the epipolar geometry constraint, they provided a truly event-based approach for real-time stereo matching. Kogler et al. proposed area-based and feature-based stereo matching algorithms for stereo DVS. ${ }^{(49)}$ They also proposed an event time-based stereo matching algorithm and showed that the time-based algorithm has a superior performance over previously proposed area-based and feature-based methods. ${ }^{(50)}$

\subsection{Control system}

Delbruck and Patrick proposed a soccer goalie robot as an example of the application of a hybrid neuromorphic-procedural system consisting of a monocular DVS camera, a computer, and a servo motor controller. ${ }^{(51)}$ Moving balls that approach the goal are tracked by an event-driven cluster tracker algorithm that was proposed by Litzenberger et al. ${ }^{(26)}$ The ball position and velocity are used to control the servo motor. The goalie robot can block balls even when they are low-contrast white-on-gray objects and there are many background distracters.

Conradt et al. proposed a pencil balancing control system where a pair of spike-based silicon retina DVSs provide fast visual feedback. ${ }^{(52)}$ This application requires very fast feedback control, successfully proving the markedly high measurement rate and low latency capabilities of the event camera. Each DVS updates its estimate of the pencil location, and the linear PD controller is used for maintaining the pencil balanced upright. Figure 10 shows the proposed pencil balancing control system hardware.

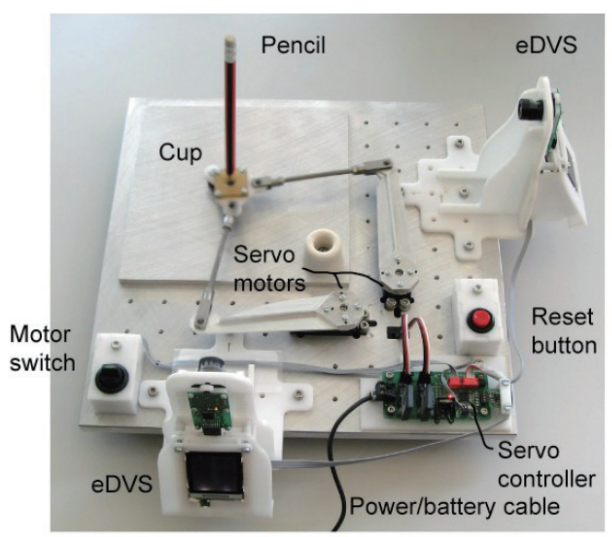

Fig. 10. (Color online) Photograph of pencil balancing control system: 2 DVSs (right top and bottom left) and the motion table actuated by two servos (center). ${ }^{(52)}$ 


\subsection{Summary of bioinspired vision sensor-based applications}

Table 2 shows a summary of the bioinspired vision sensor-based applications reviewed in this paper. Clearly, there are several advantages of DVS cameras over conventional frame-based sensors with respect to high temporal resolution, low computational power requirement due to inherent data compression, wide dynamic range, low power consumption, and wide dynamic range. In the fields of visual tracking and control using fast visual feedback, a DVS camera can be a good alternative to the conventional frame-based camera considering the speed and computational load. However, the limitations of the DVS camera are that it does not provide the absolute pixel intensity of the scene and there is no output data when the scene is static and the camera is fixed. In recognition-related fields, this can be a severe drawback because the feature extraction for classification can be strictly limited. Additionally, in SLAM and $3 \mathrm{D}$ reconstruction-related fields, feature matching and loop closure detection can be difficult with only event data. The situation is the same for ATIS cameras because a descriptor of a visually salient feature usually requires neighboring pixel information. To solve this problem, several attempts have been made to combine an event camera with an extra full frame camera. Although these are certainly possible practical solutions, these methods require an external camera system alongside the event camera, causing an increase in cost. In our view, the DAVIS camera can be a solution for this problem. The combined static and dynamic output of the DAVIS makes it promising for various applications. The DVS output can be used for tracking or segmenting the moving objects, while the image frames can be used for recognition, feature extraction, and classification. Progress in computer vision and real-time robotics using these bioinspired vision sensors is expected.

Table 2

Summary of bioinspired vision sensor-based applications.

\begin{tabular}{|c|c|c|}
\hline Category & Detail & Vision sensor setup \\
\hline \multirow{3}{*}{ Visual tracking } & Object tracking ${ }^{(26)}$ & Monocular DVS \\
\hline & Vehicle speed estimation ${ }^{(28,29)}$ & Monocular DVS \\
\hline & Optical flow estimation ${ }^{(30,32)}$ & Monocular DVS \\
\hline \multirow{2}{*}{ Detection and recognition } & Fall detection for elderly people ${ }^{(33)}$ & Monocular DVS \\
\hline & Texture recognition ${ }^{(36)}$ & Binocular DVS \\
\hline \multirow{4}{*}{ SLAM } & 2D SLAM ${ }^{(38)}$ & Monocular DVS \\
\hline & 6-DOF pose estimation ${ }^{(40)}$ & Monocular DVS \\
\hline & 3D SLAM ${ }^{(41)}$ & Monocular DVS + RGB-D camera \\
\hline & Visual odometry ${ }^{(42)}$ & Monocular DVS + CMOS camera \\
\hline \multirow{2}{*}{ Visual reconstruction } & Image reconstruction $^{(44)}$ & Monocular DVS \\
\hline & $3 \mathrm{D}$ reconstruction ${ }^{(45)}$ & $N$-ocular DVS \\
\hline Stereo matching & Stereo matching algorithm ${ }^{(47-50)}$ & Binocular DVS \\
\hline \multirow{2}{*}{ Control system } & Soccer goalie robot ${ }^{(51)}$ & Monocular DVS \\
\hline & Pencil balancer(52) & Binocular DVS \\
\hline
\end{tabular}




\section{Conclusions}

In this paper, we reviewed bioinspired vision sensors and their applications in the fields of computer vision and robotics. The reviewed bioinspired vision sensors have several advantages over conventional vision sensors, including inherent redundancy suppression, efficient in-sensor processing, fast sensing capability, wide dynamic range, and low power consumption. Until now, most of the applications and algorithms have been based on the DVS camera. The visual tracking and control-related algorithms and applications are successful cases. However, in SLAM, 3D reconstruction, and recognition-related fields, the DVS camera-based system has limitations because it does not provide any pixel intensity information. The DAVIS camera can be a good solution for this problem. In the future, much more progress in bioinspired vision sensors and their various applications in many different fields is expected.

\section{Acknowledgements}

This research was supported by a grant to the Biomimetic Robot Research Center funded by the Defense Acquisition Program Administration (UD130070ID). Editorial expenses were supported by the Brain Korea 21 Plus Project funded by the National Research Foundation of Korea (NRF).

\section{References}

1 W. S. Boyle and E. S. George: Bell Syst. Tech. J. 49 (1970) 587.

2 A. J. Theuwissen: Solid-State Imaging with Charge-Coupled Devices, ed. D. Reidel (Kluwer Academic Publishers, Dordrecht, 1995) Chap. 1.

3 D. X. Yang, A. E. Gamal, B. Fowler and H. Tian: IEEE J. Solid-State Circuits 34 (1999) 1821.

4 A. Gamal and E. Helmy: IEEE Circuits Devices Mag. 21 (2005) 6.

5 C. Posch: J. Instrum. 7 (2012) C01054.

6 C. Mead: Proc. IEEE 78 (1990) 1629.

7 C. Mead and I. Mohammed: Analog VLSI Implementation of Neural Systems (Kluwer Academic Publishers, Massachusetts, 1989) Chaps. 7-10.

8 M. Maher, S. Deweerth, M. Mahowald and C. Mead: IEEE Trans. Circuits Syst. 36 (1989) 643.

9 R. Etienne-Cummings and J. V. Spiegel: Sens. Actuators, A 56 (1996) 19.

10 G. Indiveri and D. Rodney: Science 288 (1990) 1189.

11 A. Moini: Vision Chips (Kluwer Academic Publishers, Massachusetts, 2000) Chaps. 4-9.

12 P. Lichtsteiner, C. Posch and T. Delbruck: Proc. IEEE International Solid State Circuits Conference (2006) p. 2060.

13 P. Lichtsteiner, C. Posch and T. Delbruck: IEEE J. Solid-State Circuits 43 (2008) 566.

14 C. Posch, M. Daniel and W. Rainer: Proc. IEEE International Symposium on Circuits and Systems (2008) p. 2130. 
15 C. Posch, M. Daniel and W. Rainer: IEEE J. Solid-State Circuits 46 (2011) 259.

16 C. Posch, D. Matolin and R. Wohlgenannt: Proc. IEEE International Solid State Circuits Conference (2010) p. 400.

17 C. Brandli, R. Berner, M. Yang, S. C. Liu and T. Delbruck: IEEE J. Solid-State Circuits 49 (2014) 2333.

18 R. Berner, C. Brandli, S. C. Liu and T. Delbruck: Proc. VLSI Circuits (2013) C186.

19 H. Kolb, F. Eduardo and N. Ralph: The Organization of The Retina and Visual System (Univ. of Utah Health Sciences Center, Salt Lake City, 1995) Chap. 5.

20 A. H. C. Van Der Heijiden: Selective Attention in Vision (Routledge, New York, 1992) Chap. 7.

21 J. Kramer: IEEE Trans. Circuits Syst. 49 (2002) 612.

22 K. A. Zaghloul and K. Boahen: IEEE Trans. Biomed. Eng. 51 (2004) 657.

23 M. Sivilotti: Wiring Consideration in Analog VLSI Systems with Application to Field Programmable Networks (Ph.D. thesis, 1991).

24 M. Mahowald: VLSI Analogs of Neuronal Visual Processing: a Synthesis of Form and Function (Ph.D. thesis, 1992).

25 Austrian Institute of Technology: Homepage of Austrian Institute of Technology, http://www. ait.ac.at/ (accessed on Nov. 2014).

26 M. Litzenberger, C. Posch, D. Bauer, A. N. Belbachir, P. Schon, B. Kohn and H. Garn: Proc. Digital Signal Processing Workshop (2006) p. 173.

27 D. Comaniciu and V. Ramesh: Proc. International Conference on Image Processing (2000) p. 70.

28 M. Litzenberger, A. N. Belbachir, N. Donath, G. Gritsch, H. Garn, B. Kohn, C. Posch and S. Schraml: Proc. IEEE Intelligent Transportation Systems (2006) p. 653.

29 D. Bauer, A. N. Belbachir, N. Donath, G. Gritsch, B. Kohn, M. Litzenberger, C. Posch, P. Schon and S. Schraml: EURASIP J. Embedded Syst. 1 (2007) 34.

30 R. Benosman, S. H. Leng, C. Clercq, C. Bartolozzi and M. Srinivasan: Neural Networks 27 (2012) 32 .

31 J. L. Barron, D. J. Fleet and S. S. Beauchemin: Int. J. Comput. Vision 12 (1994) 43.

32 R. Benosman, C. Clercq, X. Lagorce, S. H. Ieng and C. Bartolozzi: Neural Networks Learn. Syst. 25 (2014) 407.

33 M. Humenberger, S. Schraml, C. Sulzbachner, A. N. Belbachir, A. Srp and F. Vajda: Proc. IEEE International Conference on Computer Vision and Pattern Recognition (2012) p. 60.

34 A. N. Belbachir, M. Litzenberger, S. Schraml, M. Hofstatter, D. Bauer, P. Schon, M. Humenberger, C. Sulzbachner, T. Lunden and M. Merne: Proc. International Symposium on Circuits and Systems (2012) p. 731.

35 A. N. Belbachir, A. Nowakowska, S. Schraml, G. Wiesmann and R. Sablatnig: Proc. International Conference on Computer Vision (2011) p. 1570.

36 J. A. Pérez-Carrasco, B. Acha, C. Serrano, L. Camunas-Mesa and T. Serrano-Gotarredona: IEEE Trans. Neural Networks 21 (2010) 609.

37 B. S. Manjunath and W. Y. Ma: IEEE Trans. Pattern Anal. Mach. Intell. 18 (1996) 837.

38 D. Weikersdorfer, H. Raoul and C. Jörg: Comput. Vision Syst. 7963 (2013) 133.

39 D. Weikersdorfer and C. Jörg: Proc. IEEE International Conference on Robotics and Biomimetics (2012) p. 866.

40 E. Mueggler, H. Basil and S. Davide: Proc. IEEE/RSJ International Conference on Intelligent Robots and Systems (2014) p. 2761.

41 D. Weikersdorfer, D. B. Adrian, D. Cremers and J. Conradt: Proc. IEEE International Conference on Robotics and Automation (2014) p. 359.

42 A. Censi and S. Davide: Proc. IEEE International Conference on Robotics and Automation (2014) p. 703. 
43 R. A. Newcombe, S. Izadi, O. Hilliges, D. Molyneaus, D. Kim, A. J. Davison, P. Kohi, J. Shotton, S. Hodges and A. Fitzgibbon: Proc. IEEE International Symposium on Mixed and Augmented Reality (2011) p. 127.

44 H. Kim, A. Handa, R. Benosman, S. H. Ieng and A. J. Davison: Proc. British Machine Vision Conference (2014) p. 566.

45 J. Carneiro, S. H. Ieng, C. Posch and R. Benosman: Neural Networks 45 (2013) 27.

46 R. Benosman, S. H. Ieng, P. Rogister and C. Posch: IEEE Trans. Neural Networks 22 (2011) 1723.

47 C. Sulzbachner, K. Jürgen and E. Florian: Proc. IEEE International Symposium on ELMAR (2010) p. 467.

48 P. Rogister, R. Benosman, S. H. Ieng, P. Lichtsteiner and T. Delbruck: IEEE Trans. Neural Networks Learn. Syst. 23 (2012) 347.

49 J. Kogler, S. Christoph and K. Wilfried: Comput. Vision Syst. 5815 (2011) 174.

50 J. Kogler, H. Martin and S. Christoph: Adv. Visual Comput. 6938 (2011) 674.

51 T. Delbruck and L. Patrick: Proc. International Symposium on Circuits and Systems (2007) p. 845.

52 J. Conradt, R. Berner, M. Cook and T. Delbruck: Proc. International Conference on Computer Vision (2009) p. 780.

About the Authors

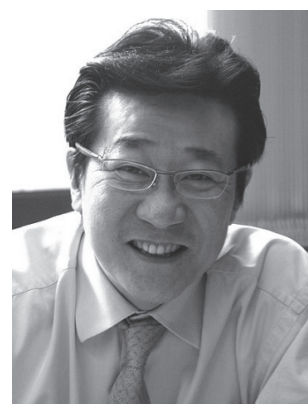

Dong-il "Dan" Cho received his B.S. degree from Carnegie Mellon University, Pittsburgh, PA, USA, in 1980, and his M.S. and Ph.D. degrees from the Massachusetts Institute of Technology, Cambridge, MA, USA, in 1984 and 1988, respectively. From 1987 to 1993, he was an Assistant Professor at Princeton University, Princeton, NJ, USA. Since 1993, he has been with the Department of Electrical and Computer Engineering, Seoul National University, Seoul, Korea, as a Professor, where he is also the Director of the Biomimetic Robot Research Center. His research interests are in MEMS, sensors, controls, and robotics. His active research and development resulted in over 300 scientific articles in English and over 100 patents. He served as a member of the International Federation of Accountants (IFAC) Technical Board from 2002 to 2005 and as the Vice Chair of the IFAC Technical Board from 2008 to 2014. Since 2014, he has been a Council Member of IFAC. He has served as an Editor and a Senior Editor of the IEEE Journal of Microelectromechanical Systems since 1992 and 2012, respectively. He was also a founding Editorial Board Member of the IOP Journal of Micromechanics and Microengineering from 1991 to 1996. He served as the President of the MEMS Research Association of Korea from 2003 to 2007. He is also a member of the International Steering Committee of Transducers from 2013 to 2021. He was inducted into the National Academy of Engineering of Korea in 2009, where he currently holds the highest membership level. 


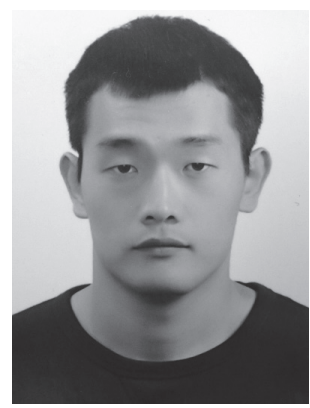

Tae-jae Lee received his B.S. degree in Electrical and Computer Engineering from Seoul National University, Seoul, Korea, in 2011. He is currently a Ph.D. candidate in the Electrical and Computer Engineering Department of Seoul National University. His research interests include robot vision, SLAM, and multisensor fusion. 